\title{
¿POR QUÉ NECESITA EUROPA UNA CONSTITUCIÓN?*
}

\author{
JÜRGEN HABERMAS
}

In this article, Jürgen Habermas addresses the role a European Constitution can play in the process of European integration. After considering the reasons that motivated integration, the author suggests that economic interest, which has been a powerful reason, will not be sufficient to mobilize political support for the project of a true political union. What can mobilize Europeans, Habermas says, is appealing to emotions rather than interests, and this could be done by gathering people to defend their way of life from the kind of society that is promoted by dominant economic models. A constitutional debate, aroused by a popular referendum, could seize the imagination of Europeans and give a cultural thrust to integration. Habermas presents the Eurosceptic argument that a European Constitution would not work because «there is as yet no European people,» and refutes this argument by distinguishing between a «nation of citizens» and an ethnic «nation of the people.» He finishes with three elements that are important for integration: a strong European Parliament, a European public sphere, and a shared political culture.

Se da un peculiar contraste entre las expectativas y demandas de los europeos de la «primera hora» quienes, apenas finalizada la Segunda Guerra Mundial, apostaron por la unión política de Europa y delinearon su proyecto, y las de aquellos otros que se enfrentan a la tarea de continuar ese proyecto ya en camino.

\footnotetext{
*Conferencia pronunciada el 26 de Junio de 2001 en el marco de la octava Hamburg Lecture, una serie de discusiones organizada conjuntamente por la Universidad de Hamburgo, el Primer Alcalde de la Ciudad Libre y Hanseática de Hamburgo, y la Fundación ZEIT Ebelin y Gerd Bucerius. Precedente agradece a la dirección de Diálogo Científico, Revista semestral de Investigaciones Alemanas sobre sociedad, derecho y economía (publicación del Zentrum für Wissenschaftiche Kommunikation mit Ibero-Amerika en colaboración con Inter Nationes Goethe Institut, Munich) la amable autorización para reproducir este artículo, aparecido en el vol. 10, № 1/2, 2001.
} 
Aquí llama la atención no sólo la diferencia de tonalidades retóricas, sino también el contraste en los objetivos. Mientras que la generación de los pioneros hablaba abiertamente de los «Estados Unidos de Europa» y no reparaba en compararlos con los Estados Unidos de América, la discusión actual se ha independizado de tales modelos. Incluso el mismo término «federalismo» es objeto de escándalo. Podemos preguntarnos si este cambio del clima político es sólo expresión de un sano realismo -como resultado de un proceso de decenios- o, por el contrario, de un contraproducente desaliento o incluso de un simple derrotismo.

Nuestra actual situación europea no es comparable a la de los federalists de antaño o a la de los miembros de la Assamblée nationale. A fines del siglo XVIII, los padres constitucionales en Filadelfia y los ciudadanos revolucionarios en París fueron iniciadores y partícipes de una praxis inaudita hasta entonces jamás vista en el mundo. Tras dos siglos de praxis constitucionalista no sólo caminamos por senderos bien pisados, sino que la pregunta por la constitución tampoco es ya la clave del problema pendiente de solución. Más aún: el desafío ya no es inventar algo nuevo sino mantener, en un formato distinto, los grandes logros del Estado nacional europeo más allá de sus fronteras nacionales: lo único aquí nuevo es la entidad que va a surgir por esa vía.

Se habrán de conservar las condiciones materiales de la vida, las oportunidades de educación y de ocio productivo, los espacios creativos sociales, los únicos en otorgar su valor útil a la autonomía privada y que hacen así posible la participación democrática. Por otro lado, no deberíamos subvalorar el peso simbólico de la circunstancia de que entretanto se haya iniciado un debate en torno a la constitución. En cuanto ente político común, Europa no puede quedar arraigada en la conciencia de sus ciudadanos sólo bajo la forma del euro. Y al acuerdo intergubernamental de Maastricht le falta aquella pujanza necesaria para consolidarse en símbolo, una pujanza que sólo puede encarnar un acto político fundacional

Hasta la generación de Helmut Kohl, la mayor fuerza impulsora hacia la integración era el deseo de poner fin a la historia de las guerras sangrientas en Europa. Otro motivo adicional, también compartido por Adenauer, era una integración de Alemania que debía suavizar la desconfianza, históricamente justificada, frente a esta nación en el centro de Europa, políticamente lábil pero económicamente de nuevo fortalecida.

Pese a que hoy todas las partes están convencidas de que definitivamente se ha logrado el primero de estos dos objetivos, la meta del aseguramiento de la paz sobrevive en un contexto totalmente distinto. En el curso de la guerra de Kosovo ha quedado clara una sutil diferencia en lo que respecta a la justificación de la intervención humanitaria. Los EE UU y Gran Bretaña consideraban las operaciones de la OTAN desde la perspectiva de sus preferencias nacionales, ampliadas con objetivos propios de la política de derechos humanos. Por el contrario, 
los Estados continentales parecían orientarse más a la luz de los principios de un futuro derecho mundial de los ciudadanos, que por la actualidad de las necesidades de orden político tal como éstas se presentan a una superpotencia que piensa a escala global.

Respecto a las transformaciones estructurales de las relaciones internacionales existen buenas razones para que la Unión Europea, apoyada en un ejército propio, hable con una sola voz en cuestiones de política exterior y de seguridad, a fin de que sus propias concepciones sean más consideradas en la OTAN y en el Consejo de Seguridad de la ONU.

La otra meta, la integración de una Alemania vista con recelo dentro de una pacífica Europa, puede que haya perdido plausibilidad en nuestro país si consideramos sus sólidas instituciones democráticas y las difundidas mentalidades liberales. Sin embargo, la reunificación de este pueblo de 82 millones de habitantes ha reavivado viejos temores de una eventual recaída en los sueños y tradiciones imperiales del Reich alemán.

Ninguno de los dos motivos mencionados contaría hoy como razón suficiente para una integración más fuerte de Europa. Sin embargo, desde el inicio existió ya un tercer interés, directamente económico, en la unión económica europea. Desde la Comunidad del Carbón y el Acero del año 1951 y desde la fundación de la Comunidad Económica Europea y Euratom en 1958, se han ido integrando cada vez más países a través del libre intercambio de personas y bienes, de capital y de prestaciones de servicios -un proceso que se ha consumado con el establecimiento del Mercado Común y la introducción de una moneda común.

Las expectativas económicas no bastan sin embargo como motivo para movilizar en la población el apoyo político para el arriesgado proyecto de una unión que realmente mereciera tal nombre. Para ello se precisa de orientaciones valorativas comunes. Ciertamente que la legitimidad de un régimen depende también de su eficiencia. Pero innovaciones políticas tales como la creación de un Estado de Estados nacionales, necesitan la movilización política para metas que apelan no sólo a los intereses sino también a los sentimientos.

Nuevas Constituciones han sido hasta ahora respuestas históricas a situaciones de crisis. ¿Pero dónde están las crisis a las que tendrían que enfrentarse las sociedades de Europa occidental que, en su conjunto, bien pueden ser calificadas como de bienestar y pacíficas? Las sociedades en transformación de Europa oriental central, interesadas en ingresar en la Unión Europea, tienen que enfrentarse de hecho a los desafíos extremos propios de una ruptura de sistema pero su respuesta fue el retorno al estado nacional-. En estos países no se detecta ningún entusiasmo por transferir a instancias europeas esos derechos soberanos que acaban de recuperar.

En vista de la falta de motivación en ambas partes, se hace tanto más patente la insuficiencia de razones exclusivamente económicas. Éstas se han de asociar 
a ideas de índole totalmente distinta para lograr, en los Estados miembros, mayorías para un cambio del status quo político; por ejemplo, con la idea de la preservación de una específica cultura y forma de vida hoy amenazada.

La gran masa de los ciudadanos europeos se siente unida en el interés por defender una forma de vida que ellos pudieron desarrollar en las regiones favorecidas a este lado de la «cortina de hierro» durante el segundo tercio del siglo pasado, o sea, en «los años dorados», según Eric J. Hobsbawm ${ }^{1}$. Sin duda, un rápido crecimiento económico echó los fundamentos de un Estado social en cuyo marco se regeneraron las sociedades europeas de postguerra. Pero como resultado de esa generación sólo cuenta una cosa: modos de vida en los que sobre la base del bienestar y de la seguridad se ha ido diferenciando la riqueza y la diversidad nacional de una cultura atractivamente renovada que lleva siglos de antigüedad.

Las ventajas económicas de la unificación europea cuentan como argumento para una mayor ampliación de la UE solo en el contexto de un fuerte atractivo cultural que va mucho más allá de la dimensión económica. Las amenazas para esta forma de vida, y el deseo de preservarla, estimulan la visión de una futura Europa dispuesta a aceptar de nuevo, innovativamente, los desafíos actuales.

El primer ministro francés, en su grandioso discurso del pasado 28 de Mayo, ha señalado este «modo de vida europeo» como contenido del proyecto político: «Hasta hace poco, los esfuerzos de la Unión se concentraban en la creación de la unión monetaria y económica..., pero hoy se precisa de una perspectiva más amplia, de lo contrario Europa degenerará en un mero mercado y se ablandará en la globalización. Porque Europa es mucho más que un mercado. Europa representa un modelo de sociedad que ha ido creciendo en la historia.»

¿Pero pueden nuestros pequeños o medianos Estados nacionales, por sí solos, mantener la capacidad de acción para resistir al destino de una subrepticia asimilación al modelo de sociedad que les insinúa el régimen económico hoy reinante en el mundo? Este modelo, en términos polémicos, está marcado por cuatro momentos:

- por la imagen antropológica del ser humano como un empresario que decide racionalmente, que explota su propia fuerza de trabajo;

- por la imagen social moral de una sociedad postigualitaria que se resigna a la existencia de marginalizaciones, recusaciones y exclusiones;

\footnotetext{
${ }_{1}^{1}$ «Los años dorados» es el título del capítulo IX del libro de Hobsbawm, Historia del siglo XX, Buenos Aires, Crítica Grijalbo Mondadori, 1998 (Nota de Precedente).
} 
- por la imagen económica de una democracia que reduce a los ciudadanos de un Estado a la condición de miembros de una sociedad de mercado y redefine al Estado como empresa de prestación de servicios para clientes y parroquianos;

- finalmente, por la pretensión estratégica de que no existe mejor política que la practicada por él mismo.

Estas son piezas de una imagen neoliberal del mundo que, si no me equivoco, no se adapta bien a la concepción normativa que hasta ahora los europeos tenían predominantemente de sí. ¿Qué lectura del proyecto de unificación europea insinúa este diagnóstico? En la medida en que los europeos quieran balancear las indeseables consecuencias sociales de crecientes desigualdades distributivas, e influir en una cierta renovada regularización de la economía mundial, tendrán también que tener un interés en el potencial constitutivo que lograría una Unión Europea capaz de ejercicio político en el círculo de los global players.

El actual debate sobre la reforma ha sido provocado por el dilema de la «crisis de la ampliación». Con la fecha para el ingreso de los Estados del este europeo, la UE misma se ha sometido a la presión de una reforma. La ampliación de la Unión a doce países, en términos económicos y sociales relativamente heterogéneos, incrementa la complejidad de una necesidad de regulación y sintonización que no puede ser resuelta sin una ulterior integración o «profundización».

La Conferencia de Niza no ha resuelto este atasco reformista. No se ha logrado convertir el actual problema de la ampliación en la palanca para la elaboración de problemas estructurales con base más profunda. Aquí se trata, por un lado, del desequilibrio entre la imbricación económica y la política densa la primera y más bien relajada la segunda- y, por otro, del déficit democrático de los procesos de decisión de Bruselas.

La discrepancia entre la avanzada integración económica y la retardada integración política podría ser superada por una política que apuntara a la construcción de capacidades políticas de acción en un nivel más alto, ya que quiere guardar el paso con los mercados desregularizados. Desde esta perspectiva, el proyecto europeo aparece como el intento común de los gobiernos nacionales de recuperar en Bruselas algo de la capacidad de intervención que cada uno de ellos ha perdido en casa. Así lo ve al menos Lionel Jospin que exige para la eurozona un gobierno económico así como, a largo plazo, una armonización de toda la imposición fiscal a las empresas.

La coordinación de más ámbitos de la política significa naturalmente una concentración de competencias, la cual hace todavía más agudo un dilema. La densidad de decisiones europeas, la impenetrabilidad de su gestión y la faltante oportunidad para los ciudadanos europeos de participar en los procesos de decisión, provocan desconfianza en la base. 
Claus Offe ha estudiado los temas y los complejos necesitados de regulación que despiertan temores en cada una de las naciones y provocan rivalidades entre ellas. Las preocupaciones apuntan en primera línea a los efectos fiscales de redistribución, que podrían suponer una desventaja para los propios compatriotas y un beneficio para otras naciones. También despiertan temores las corrientes migratorias desde países extraños, y las corrientes inversoras que fluyen hacia países extraños. Offe describe la actual situación de las relaciones entre las naciones que pertenecen a la UE como un «estado natural pacífico». Tal estado sólo podría ser superado, según él, con una «formación de Estados» europea, pero que no debería orientarse por el patrón del Estado nacional.

Los euroescépticos rechazan un cambio de la base de legitimación de acuerdos internacionales hacia una constitución europea, argumentando que «no existe un pueblo europeo», tal como escribe el ex juez constitucional ErnstWolfgang Böckenförde. Lo que parece faltar es el debido sujeto de un proceso constituyente, es decir, ese singular colectivo del «pueblo» que podría constituirse a sí mismo como una nación de ciudadanos.

Esta «no-demos-thesis» ha sido criticada por razones conceptuales y empíricas. La nación de los ciudadanos no debe ser confundida con una comunidad de destino pre-política, marcada por un origen, lengua e historia comunes, puesto que con ello se erraría el carácter voluntarista de una nación de ciudadanos, cuya identidad colectiva no existe, ni antes ni en absoluto, independientemente del proceso democrático del que ella surge.

En este contraste de nación de ciudadanos y nación de pueblo se refleja el gran logro del Estado nacional democrático que con el status de la ciudadanía ha generado por primera vez una solidaridad totalmente nueva, una solidaridad abstracta, mediada a base del derecho.

Aun cuando la lengua y forma de vida comunes han facilitado este proceso de formación de una conciencia, no se puede deducir de la circunstancia de que democracia y Estado nacional se hayan desarrollado al mismo paso, que el pueblo tenga la prioridad frente a la república. Más bien se trató de un proceso circular en cuyo curso fueron estabilizándose recíprocamente conciencia nacional y ciudadanía democrática. Ambas han dado lugar conjuntamente al nuevo fenómeno de una solidaridad entre ciudadanos que desde entonces constituye el aglutinante de las sociedades nacionales.

A raíz de esta historia de la génesis de los Estados nacionales europeos, se puede aprender que las nuevas formas de la identidad nacional tienen un carácter artificial que se ha ido formando bajo determinados condicionantes históricos en el curso de un largo proceso que se extiende por todo el siglo XIX. Esta formación de identidad se debe a un doloroso proceso de abstracción, que ha llevado desde las identiudades locales y dinásticas a las identi- 
dades nacionales y democráticas. Si esto es así, no hay entonces razón alguna para suponer que la formación de un tal tipo de solidaridad ciudadana habría de detenerse en las fronteras del Estado nacional.

Las condiciones bajo las que surgió la conciencia de nación nos recuerdan por lo demás los requisitos empíricos que han de ser cumplidos para que una formación de identidad tan improbable pueda difundirse también más allá de las frontera nacionales: en primer lugar, la necesidad de una sociedad europea de ciudadanos; en segundo lugar, la construcción de una esfera pública política en toda Europa; y, tercero, la creación de una cultura política que pueda ser compartida por todos los ciudadanos de la UE.

Estos tres requisitos funcionales de una Unión Europea democráticamente constituida se pueden entender como puntos de referencia de desarrollos complejos pero convergentes. Estos procesos pueden ser acelerados a través de una constitución que tiene un efecto en cierto modo catalizador, y ser dirigidos hacia el punto de convergencia. Por así decir, Europa tiene que volver a aplicarse reflexivamente a sí misma la lógica de aquel proceso circular en el que se generaron recíprocamente el Estado democrático y la nación. En los inicios estaría un referendo sobre la constitución, que pondría en marcha un gran debate en toda Europa. El proceso constituyente es ya en sí un peculiar medio de comunicación allende las fronteras. Tiene el potencial de una selffulfilling prophecy. Una constitución europea no sólo haría manifiesto el desplazamiento del poder que, tácitamente, ya ha tenido lugar; también promovería nuevas constelaciones de poder.

Primero: una vez que la Unión Europea se hiciera financieramente autónoma, la Comisión asumiera las funciones de un gobierno y el Consejo llegara a ser como una segunda cámara, el Parlamento Europeo atraería mayor atención hacia un ejercicio mejor establecido, y más visible, de competencias que ya ahora son notables. Para ello ni siquiera se precisa, inicialmente, la plena soberanía presupuestaria de las clásicas representaciones populares.

El eje de la política se inclinaría más desde las capitales nacionales hacia Bruselas y Estrasburgo. Además, los intereses organizados de acuerdo al sector económico y grupo profesional, a la confesión religiosa e ideología política, a la clase, región y sexo, se fusionarían por encima de las fronteras nacionales. La perceptible superposición transnacional de intereses paralelos fomentaría el surgimiento de un sistema de partidos europeos y de redes allende las fronteras. De esta manera, las formas territoriales de la organización serían convertidas en principios funcionales, de modo que surgirían condiciones de asociación que podrían formar el núcleo de una sociedad civil en toda Europa.

Segundo: no habría remedio para el deficit de legitimación, sin embargo, sin la existencia de una vasta esfera pública europea, es decir, sin una red que diera a todos los ciudadanos de los Estados miembros igual oportunidad de 
tomar parte en un proceso incluyente de comunicación política. En sociedades complejas, la legitimación democrática requiere un contacto recíproco de procesos de deliberación institucionalizada y de toma de decisiones en los parlamentos, cortes e instancias administrativas -por un lado-, y de un proceso abarcador de comunicación informal masiva. Ocurre que tales espacios de formación pública de opinión y voluntad, de momento sólo se dan dentro de algunos Estados nacionales. Pero no hay que imaginarse la vasta esfera pública europea como la proyección de un diseño familiar que se desplaza desde lo nacional hacia un nivel europeo. Ella habrá de surgir más bien de una apertura recíproca de los existentes universos nacionales que dé lugar a una interpenetración de comunicaciones nacionales que se trasladan con reciprocidad. Los medios nacionales de un país habrán de recoger y comentar la sustancia de las controversias que tienen lugar en otros Estados miembros. Así será posible que en todos los países se vayan formando opiniones paralelas, a favor y en contra, sobre un mismo tipo de objetos, informaciones y razones, independientemente de cuál sea su procedencia. El hecho de que las comunicaciones que aquí fluyen horizontalmente de un lado a otro hayan de pasar por el filtro de las traducciones recíprocas no merma su significación esencial.

Tercero: la generación de una opinión pública europea depende de las intervenciones vitales de actores de la sociedad civil. Al mismo tiempo, una vasta esfera pública europea necesita también de estar enmarcada dentro de una cultura política compartida por todos. Esta exigencia vastamente percibida ha estimulado un preocupado debate entre los intelectuales, debido a que ha sido difícil separar la pregunta «¿Qué es Europa?» del hecho de que las realizaciones de la cultura europea -que, de hecho, no reflexionó sobre sí misma hasta los siglos XVIII y XIX- se han difundido por todo el globo. El cristianis232 mo, la ciencia moderna y la tecnología, el derecho romano y el Código de Napoleón, el Estado nacional, la democracia y los derechos humanos se difundieron por todo el mundo desde Europa. Mencionemos además dos experiencias específicas de nuestros países que han tenido un eco digno de atención. En su historia, Europa ha sido confrontada más que otras culturas con conflictos y tensiones de profundas raíces y estructuralmente afianzados, y ello tanto en lo que concierne a la dimensión social como a la temporal. De ahí se explica sin duda también su agresiva disponibilidad a la expansión y su alto potencial de violencia. Pero los europeos también han reaccionado productivamente ante tales desafíos y aprendido en ello sobre todo dos cosas: a vivir con conflictos duraderos estabilizados, y a adoptar una postura reflexiva frente a sus propias tradiciones.

En la dimensión social, la Europa moderna ha desarrollado procedimientos e instituciones para el tratamiento de conflictos intelectuales, sociales y 
políticos. En el curso de dolorosos y con frecuencia fatales conflictos, Europa ha aprendido a valérselas en medio de la competencia entre poderes religiosos y seculares, de la separación entre fe y conocimiento, de la endémica contienda entre las confesiones y, al final, también de la hostilidad y rivalidad entre belicosos Estados nacionales. Esto lo hemos logrado no por haber solucionado tales conflictos, sino por haberlos perpetuado a través de la ritualización, y convertido en fuente de energías innovadoras.

A las fracturas, discontinuidades y tensiones experimentadas en la dimensión temporal, inherentes a todo proceso de modernización, la Europa de la Revolución francesa respondió con el establecimiento de un certamen ideológico entre los partidos políticos. El clásico sistema de partidos cuida de la reproducción de un amplio espectro de interpretaciones conservadoras, liberales y socialistas de la modernización capitalista. Como consecuencia de la heroica apropiación intelectual de un patrimonio judío y griego, romano y cristiano, incomparablemente rico, Europa ha aprendido cómo adoptar repetidamente una postura sensible frente al rostro jánico de la modernidad.

En todo caso, el universalismo igualitario e individualista que hasta hoy marca nuestra autocomprensión normativa, no es la más ínfima entre las conquistas de la modernidad europea. El hecho de que la pena de muerte siga siendo practicada en otros lugares, hasta en los Estados Unidos, nos hace recordar rasgos específicos de nuestra propia conciencia normativa.

Lo que integra el núcleo de la identidad europea es naturalmente más el carácter de dolorosos procesos que su resultado. El recuerdo del abismo moral a que nos llevó el exceso nacionalsocialista, confiere a nuestro actual compromiso el rango de una conquista. Este trasfondo histórico podría allanar la transición hacia una democracia postnacional apoyada en el reconocimiento recíproco de las diferencias entre orgullosas culturas nacionales. $\mathrm{Ni}$ «asimilación» ni mera «coexistencia» (en el sentido de un tambaleante modus vivendi) son los modelos adecuados a esta historia - una historia que nos ha enseñado cómo poder establecer siempre formas más abstractas de una «solidaridad entre extraños».

La población que en su mavoría tiene una actitud de rechazo o, al menos, vacilante, podrá ser ganada para Europa sólo si el proyecto es disociado de la descolorida abstracción de las medidas administrativas y conversaciones entre expertos, es decir, si el proyecto es politizado. Los intelectuales no han recogido la pelota, y los políticos, muy en especial, tampoco quieren quemarse los dedos al tocar un tema poco popular.

Tanto más notable es por ello que el discurso de Joschka Fischer en la Universidad Humboldt de Berlín (12 de Mayo de 2000) haya significado un empujón hacia un debate sobre la constitución. A una pregunta sobre cómo 
establecer el enlace correcto entre la Europa de los Estados y la Europa de los ciudadanos, han reaccionado con propuestas propias Chirac y Prodi, Rau y Schröder. Pero ha sido Jospin quien ha dejado bien claro que una reforma de procedimientos e instituciones no logrará su objetivo antes de que el contenido del proyecto político tenga perfiles más precisos. 